\title{
Impact of Clustering in Indoor MIMO Propagation Using a Hybrid Channel Model
}

\author{
Zhongwei Tang \\ Microwave and Wireless Technology Research Laboratory, Information and Communication Group, Faculty of Engineering, \\ University of Technology, Sydney, L24/B1, P.O. Box 123, Broadway NSW 2007, Australia \\ Email: zhongwei@eng.uts.edu.au
}

\begin{abstract}
Ananda Sanagavarapu Mohan
Microwave and Wireless Technology Research Laboratory, Information and Communication Group, Faculty of Engineering, University of Technology, Sydney, L24/B1, P.O. Box 123, Broadway NSW 2007, Australia

Email:ananda@eng.uts.edu.au
\end{abstract}

Received 1 March 2004; Revised 7 October 2004

\begin{abstract}
The clustering of propagating signals in indoor environments can influence the performance of multiple-input multiple-output (MIMO) systems that employ multiple-element antennas at the transmitter and receiver. In order to clarify the effect of clustering propagation on the performance of indoor MIMO systems, we propose a simple and efficient indoor MIMO channel model. The proposed model, which is validated with on-site measurements, combines the statistical characteristics of signal clusters with deterministic ray tracing approach. Using the proposed model, the effect of signal clusters and the presence of the line-of-sight component in indoor Ricean channels are studied. Simulation results on channel efficiency and the angular sensitivity for different antenna array topologies inside a specified indoor scenario are also provided. Our investigations confirm that the clustering of signals significantly affects the spatial correlation, and hence, the achievable indoor MIMO capacity.
\end{abstract}

Keywords and phrases: angle sensitivity, channel efficiency, indoor propagation, signal clusters, MIMO, Ricean $K$ factor, ray tracing.

\section{INTRODUCTION}

The multiple-input multiple-output (MIMO) technique is being tipped as one of the most significant breakthroughs in wireless communications for achieving high data-rates without increasing the channel bandwidth $[1,2,3,4]$. In view of its significance, the MIMO technique is considered for inclusion into the forthcoming IEEE 802.11n WLAN standard. MIMO systems have the ability to turn multipath propagation into a benefit for users by employing multiple antennas at both the transmitter and receiver to exploit multipath fading, in order to maximize data throughput. The underlying mathematical nature of MIMO, where the data is transmitted over a matrix rather than a vector channel, creates new and enormous opportunities beyond just diversity or array gain benefits. This has prompted new research on channel modelling, antenna design, coding schemes and signal processing, and so forth.

In MIMO systems, the channel transfer matrix is a key component that includes the coupling information between the transmitter and the receiver and their interaction with the surrounding physical environment, through the spatial and angular features of RF propagation. It has been reported that the correlation of the channel transfer matrix due to directional multipath propagation tends to decrease MIMO performance $[5,6,7]$ for both indoor and outdoor MIMO systems, when time diversity is not considered [8]. Thus, the characteristics of the transmit and receive arrays, such as antenna polarization [9], antenna element separation [10], and array topology and orientations [11] can play a major role in determining the achievable MIMO capacity. It has also been reported $[12,13]$ that the presence of the line-of-sight (LOS) component in Ricean channels also reduces the achievable MIMO capacity.

As the antenna characteristics and channel correlations affect the achievable spectrum efficiency, antenna selection assumes importance for obtaining the optimized capacity $[14,15]$. On the other hand, in indoor propagation environments, it has been well-established that multipath waves tend to be clustered in both angular and temporal domains $[16,17,18,19]$. Moreover, the clustering propagation is found to be detrimental to indoor MIMO performance as it increases the spatial correlation between subchannels $[20,21]$. When antenna arrays are employed at both the transmitter and receiver in indoor environments, the correlation between antenna elements is a function of the signal 
clusters, whose characteristics are determined by the physical features of a given indoor environment as well as the locations of the transmitter and receiver. Since antennas are key components in MIMO systems, it is important to understand the impact of antenna array topology and orientation on achievable capacity in clustering indoor environments. Many realistic indoor environments are Ricean scenarios since it is common for a strong line-of-sight component between the transmitter and receiver to exist. Thus an investigation on the effect of Ricean $K$ factor on the achievable MIMO capacity assumes practical importance. Further, the subchannel efficiency in indoor environments is not fully addressed. Therefore, a comprehensive investigation on the impact of signal clustering on indoor MIMO performance is expedient for the efficient design and deployment of highperformance wireless systems. To this end, it is essential to exploit the MIMO channel transfer matrix by including the spatial and angular behaviour of multipath clusters.

In the pioneering work of Foschini and Gans [3] and Telatar [4], the MIMO channels were modelled to be uncorrelated with the entries of the channel matrix being independent and identically distributed (i.i.d.) complex Gaussian variables with zero-mean and unit variance. However, such i.i.d. models do not represent the behaviour of real indoor correlated channels. Attempts have been made to use direct measurements to form the indoor channel transfer matrix $[6,22]$. However, such models may have limited applicability to specific array configurations or propagation environments under test. Wallace and Jensen [23] and others [16, 17] have employed measurement-based statistical models to generate the channel parameters by fitting the models to measured data. These statistical models can be quite useful in generalizing the spatial behaviour for typical indoor channels; but require extensive measurements. On the other hand, deterministic ray tracing has been applied to investigate indoor MIMO performance for simple indoor environments $[13,24]$. However, the use of rigorous $3 \mathrm{D}$ ray tracing alone to accurately model realistic indoor MIMO environments can be computationally intensive. Thus simple yet tractable MIMO channel models that can incorporate clustering propagation by exploiting the statistical features of RF propagation are desirable.

In this paper, we propose a hybrid indoor MIMO channel model to include the effect of clustering propagation on the MIMO channel matrix. The hybrid indoor MIMO channel model uses deterministic ray tracing approach to detect the effective signal clusters in a given environment, which will be combined with the stochastic features of the clusters to construct the MIMO channel matrix. The model then is used for predicting indoor MIMO characteristics such as the mutual information as a function of antenna topology, spatial correlation, and the Ricean $K$ factor for specified indoor environments. The results predicted using the hybrid cluster channel model are also validated by comparing with on-site measurements in terms of MIMO capacity. It is expected that the simulation results on the channel parameters presented in this paper may provide detailed insights on the impact of clustering propagation for the design of indoor MIMO systems.
The paper is organized as follows: Section 2 reviews the indoor clustering propagation, the statistical properties of signal clusters, as well as the ray tracing modelling approach; Section 3 introduces the signal model and the channel capacity of MIMO systems; in Section 4 an indoor MIMO hybrid channel model is proposed which combines the stochastic properties of signal clusters with the deterministic ray tracing approach; and Section 5 presents the results on the effect of clustering propagation on indoor MIMO performance obtained using the hybrid channel model. The conclusions are drawn in Section 6.

\section{INDOOR CLUSTERING PROPAGATION AND RAY TRACING MODELLING}

The propagation of RF waves in indoor environments is complex and has been studied extensively for applications of conventional indoor single-input single-output (SISO) wireless communications $[25,26]$. The clustering of multipath waves in indoor propagation was first observed by Saleh and Valenzuela [16] during their RF indoor channel measurement campaign. For a specified transmitter and receiver pair in a given indoor environment, the receive antenna receives a finite number of multipath clusters which arrive from certain directions. The individual signal cluster may consist of a large number of signal components caused by the scattering from local scatterers in the vicinity of both the transmitter and receiver. Spencer et al. [17] proposed a statistical indoor SISO channel model by including the angular characteristics of signal clusters using measured data. In this model, the angles of arrival (AOA) of signal clusters were assigned to be uniformly distributed in the range of $[0,2 \pi)$; and the Laplacian probability density function (PDF) with varying angle spreads was utilized to fit the AOAs of the multipath components (MPCs) within individual clusters. A clustering SISO model based on geometric ray bouncing in elliptical subregions was proposed in [27], in which the scatterers were assumed to be uniformly distributed within the scatter region, and the Gaussian distribution was used to characterize the angular property of signal rays within each cluster. A statistical wideband cluster model is proposed in [19] which confirms the suitability of using Laplacian PDF for representing signal clusters.

In the modelling of indoor multipath channel, the clustering phenomenon was also captured by using the deterministic ray tracing approach $[17,18]$. The deterministic ray tracing approach was traditionally employed to predict the static SISO channel characteristics, such as the narrowband received signal power, large-scale path loss, the power delay profile, and so forth, of indoor radio channels [28, 29], whose prediction accuracy has also been verified with measurements $[28,29,30]$. In this paper, we define a cluster to be an accumulation of multipath components (rays) with similar angular characteristics, such as AOA or AOD, and a ray, referring to a single wave, is interchangeable with the term multipath component. The ray tracing technique is based on geometric optic theory, and so it is possible to capture the angular information of signal rays, hence the cluster of 
a group of signal rays, using the geometry. The angular information of effective signal clusters can be determined by the relative positions and orientations of the transmitter and receiver as well as the physical features of an indoor environment. Comparisons between existing ray tracing predictions and measurements on the angular information of signal clusters, as reported in $[29,31]$, show consistent agreement between both approaches. These support the use of ray tracing in the prediction of angular information of propagating clusters in specified indoor environments.

\section{MIMO SYSTEM MODEL}

We consider a point-to-point narrowband indoor communication link with $P$ transmit and $M$ receive antennas. A flat fading channel is assumed here. This is valid if the coherence bandwidth of the channel is larger than the transmission bandwidth. The complex baseband input-output signal relationship of the narrowband MIMO system is represented as

$$
\vec{r}=H \vec{s}+\vec{n}
$$

where $\vec{r}$ is the received signal vector, $\vec{s}$ is the transmit signal vector, and the entry $h_{i j}$ of the channel transfer matrix, $H=\left[h_{i j}\right]_{M \times P}$, is the complex transfer function between the $j$ th transmit antenna and the $i$ th receive antenna, for $i=1,2, \ldots, M$ and $j=1,2, \ldots, P$. The signal is assumed to be corrupted by white Gaussian noise $\vec{n}$, which is a vector composed of independent complex Gaussian-distributed elements with a zero-mean and variance $\sigma^{2}$.

The consideration of quasistationary flat fading channels allows $H$ to be constant during one or more communication bursts, as is usually the case in indoor wireless communication implementations, where both the transmitter and receiver are of limited mobility. The Shannon channel capacity of an MIMO link is calculated as [3,4]

$$
C_{\text {Equalpower }}=\log _{2}\left(\operatorname{det}\left(I+\frac{\rho_{\mathrm{SNR}}}{P} H H^{*}\right)\right),
$$

where $\operatorname{det}(\cdot)$ denotes the determinant, $\rho_{\mathrm{SNR}}$ is the desired average received signal-to-noise ratio, $I$ is an identity matrix, $P$ is the number of transmit antennas, $H^{*}$ denotes the complex conjugate transpose of the normalized channel matrix $H$, and $C$, in bits $/ \mathrm{s} / \mathrm{Hz}$, is the mutual information. In this case, the channel state information is assumed to be available at the receiver side only, and the transmitted power is equally allocated to each transmit element.

Using singular value decomposition (SVD), the channel matrix can be decomposed as

$$
H=U \Lambda V^{*}=U \operatorname{dia}\left(\sqrt{\lambda_{1}}, \sqrt{\lambda_{2}}, \ldots, \sqrt{\lambda_{m}}, 0, \ldots, 0\right) V^{*},
$$

where $U$ and $V$ are unitary matrices whose elements are the corresponding singular vectors of the diagonal matrix $\Lambda$.
The elements of $\Lambda$ are the square roots of the eigenvalues, $\lambda_{i}$, $i \in[1, \ldots, m], m \leq \min (M, P)$, of the channel covariance matrix, $R_{H}$, and $R_{H}=H H^{*}$ for $M \leq P$ or $H^{*} H$ for $P<M$. The capacity for the equal power allocation scheme in (2) can be rewritten as [32]

$$
C_{\text {Equalpower }}=\sum_{i=1}^{m} \log _{2}\left(1+\frac{\rho_{\mathrm{SNR}} \lambda_{i}}{P}\right) .
$$

Effectively, the MIMO channel is decomposed into $m$ parallel spatial SISO subchannels, each one of which has a corresponding channel gain $\lambda_{i}$.

When the channel state information is available at both the transmitter and receiver, the capacity can then be optimized by applying the waterfilling solution with a transmit power constraint. The result in capacity is given as

$$
C_{\text {Waterfilling }}=\sum_{i=1}^{m} \log _{2} \operatorname{det}\left(1+\frac{P_{i} \lambda_{i}}{\sigma^{2}}\right)
$$

where $P_{T}=\sum P_{i}$ is the total transmit power, $P_{i}=\left(u-1 / \lambda_{i}\right)^{+}$ is the transmitted power in the $i$ th subchannel, $(\cdot)^{+}$indicates taking only those terms which are positive, and $\mu$ is called the water level.

\section{A HYBRID INDOOR MIMO CHANNEL MODEL}

The aim in modelling the MIMO channel is to obtain the channel transfer matrix $H$ that characterizes the fading of correlated multipath which couples the transmitter and the receiver. In this section, we present a hybrid indoor MIMO channel model, where a $2 \mathrm{D}$ ray tracing method is used to obtain the angular features of signal clusters for realistic indoor environments, that will be combined with the statistical properties of signal clusters to construct the MIMO channel matrix. The MIMO channel matrix is formed using the Kronecker method, which is based on the covariance matrices at the transmitter and receiver, so as to ensure that the channel matrices inherit the spatial fading correlation of a given channel. In order to obtain the correlation coefficients at both the sides, the angular information, namely, angles of departure (AOD) and angles of arrival of signal clusters, is necessary and is calculated in this paper by using an imagebased deterministic ray tracing approach. Our aim is to understand the fading caused by the spatial effect rather than the effect of temporal dispersion. Therefore, throughout our work, narrowband flat fading channels are assumed.

\subsection{The spatial correlation between antennas in clustering channels}

The spatial correlation of signals received by two adjacent antenna elements of an array describes how the received fields are correlated, which in turn determines the rank of the MIMO channel matrix. The degree of the spatial correlation depends on the interelement spacing between array elements, the radiation pattern, and the angular features of received signals. The spatial correlation for unclustered 
Rayleigh channels is well known [33], where multipath is assumed to arrive uniformly within $360^{\circ}$ around the antennas. We denote this scenario as the unclustered case. The correlation for this classic case is given by [33]

$$
\rho(d)=J_{0}\left(k_{0} d\right)
$$

where $J_{0}$ is the Bessel function of the zeroth order, $k_{0}=2 \pi / \lambda$ is the wave number, and $d$ is the separation between two antennas. However, it is well-established that uniform distribution does not properly represent the angular characteristics of multipath in indoor propagation channels where propagating waves tend to be clustered $[16,17,19]$. The physical features of indoor environments cause the rays to scatter nonisotropically. Since a cluster is an accumulation of individual rays with similar angular characteristics, it is possible for rays to form clusters nonuniformly in the angular domain. In this paper, we make use of the deterministic ray tracing only to find the number of dominant clusters, and their angular characteristics. However, to represent the nonuniform statistics of the individual rays within each cluster, we have used the Laplacian distribution.

Now, to derive the spatial correlation between two antenna elements due to signal clusters in indoor clustering channels, we first derive the spatial correlation between two adjacent antenna elements due to finite signal clusters in a non-line-of-sight (NLOS) Rayleigh scenario. The correlation for Ricean channels is later obtained by accounting for the Ricean $K$ factor. For an indoor channel where clustering propagation occurs, the assumption of local plane waves (far-field approximation) is realistic when the separation between the sources of the clustering waves and the antenna arrays are larger than the array aperture. Therefore, the direction of propagation can be approximated to be the same at each array element and the array elements are assumed to be within the same small-scale fading area and receive identical clusters. To simplify the problem, we further assume that the effective clusters are confined within the horizontal plane. Based on these assumptions, we proceed to derive the spatial correlation of received signals at two adjacent antenna elements due to an incident reference cluster.

Let $r_{1}(t)$ and $r_{2}(t)$ denote the complex envelopes of the received signals at any two adjacent antennas, and the correlation coefficient is defined as [34]

$$
\rho(d)=\frac{E\left\{r_{1}(t) r_{2}^{*}(t)\right\}}{\sqrt{E\left\{\left|r_{1}(t)\right|^{2}\right\} E\left\{\left|r_{2}(t)\right|^{2}\right\}}},
$$

where $E\{\cdot\}$ denotes the expectation operator, $*$ denotes the complex conjugate transpose operation, $d$ is the separation between the two antennas. Since the difference between the received signals at the two adjacent elements induced by an incident plane wave is mostly a phase factor, the correlation between two identical antennas due to a single wave arriving from an angle $\theta$ can be approximated as

$$
\rho(d)=e^{-j k_{0} d \cos \theta}
$$

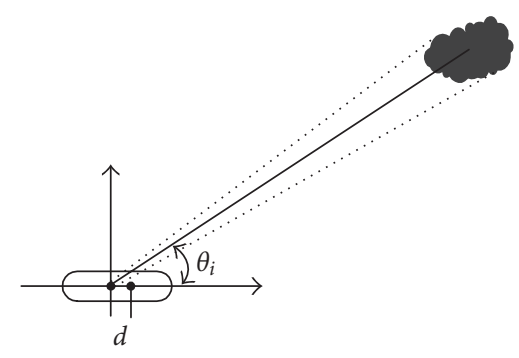

Figure 1: A reference cluster impinging on a two-element array.

where $k_{0}$ is the wave number. Now assume the reference cluster to arrive from the direction of $\theta_{i}$, as depicted in Figure 1. Since the number of rays within a cluster can be very large, the resultant correlation, $\rho\left(d, \theta_{i}\right)$, due to the reference cluster can be obtained as

$$
\rho\left(d, \theta_{i}\right)=E\left\{e^{-j k_{0} d \cos \left(\theta_{i}+\theta\right)}\right\}=\int_{\theta} e^{-j k_{0} d \cos \left(\theta_{i}+\theta\right)} p(\theta) d \theta
$$

where $\theta$ is the AOA of an individual ray within the cluster with respect to the mean, $\theta_{i}$, of the reference cluster; $p(\theta)$ is the probability density function that characterizes the angles of arrival of individual rays with respect to $\theta_{i}$.

Knowing the distribution $p(\theta)$ of the AOAs of all the multipath components within each cluster, one can obtain the spatial correlation using (9). Based on the evidence that the Laplacian distribution fits the measured signal cluster data well in typical indoor environments as reported in $[17,19]$, here we adopt a zero-mean Laplacian distribution to represent the angular features of multipath components within each cluster.

With the angular information on hand, the spatial correlation between two receiving antenna elements, $\rho_{\mathrm{NLOS}}(d)$, due to all effective signal clusters in a Rayleigh scenario, can be calculated using

$$
\rho_{\mathrm{NLOS}}(d)=E\left[\rho\left(d, \theta_{i}\right)\right]
$$

Equation (10) is suitable when there is no direct line-of-sight path between transmit and receive antennas. If a dominant LOS component exists between them as in Ricean channels, the total spatial correlation $\rho(d)$ needs to include the Ricean factor, $K$, which is given by [12]

$$
\rho(d)=\frac{K}{K+1} \rho_{\mathrm{LOS}}(d)+\frac{1}{K+1} \rho_{\mathrm{NLOS}}(d),
$$

where $\rho_{\mathrm{LOS}}(d)$ denotes the spatial correlation due to the LOS cluster in Ricean channels, which can be calculated using (8), $\rho_{\mathrm{NLOS}}(d)$ denotes the spatial correlation due to all NLOS clusters obtained using (10), and $K$ is the Ricean factor defined as the ratio between the signal powers of the LOS and all NLOS components. 


\subsection{Use of ray tracing to obtain AOAs and $A O D$ s of signal clusters}

The accuracy of ray tracing predictions of multipath in indoor radio channels has been well-established [28, 30, 31]. The results of angles of arrival of multipath components calculated using an image-based $3 \mathrm{D}$ ray tracing tool were validated with the "measured AOA" obtained by applying the spatially smoothed MUSIC algorithm on measurement data by Wang et al. [29]. The authors in [31] also reported a good agreement between the ray tracing predictions and measurements on the angular distribution of multipath waves in indoor channels. Thus the consistency with which the ray tracing predictions were validated with measurements forms the prime motivation for us to make use of ray tracing to obtain the angular characteristics of signal clusters in this paper.

When antenna arrays are used in an indoor environment at both the transmitter and receiver, the assumption of small array aperture is valid as long as $B a / c \ll 1$ holds, where $B$ is the signal bandwidth, $a$ is the size of the antenna array, and $c$ is the speed of light. Therefore, it can be approximated that all the elements of the antenna array are located within the same small-scale area with respect to the reference element of the array within which the small-scale channel properties are assumed to be identical. Accordingly, the channel transfer functions of the MIMO connection can be viewed as a realization of a random process with the same statistics, including the average power, the temporal and angular features of signal clusters. For the sake of convenience, the transmitter and receiver are assumed to be located at the same height and only the rays confined within a horizontal plane are considered in this paper.

Following [29], based on the assumption that the dominant paths are confined in the same horizontal plane as that containing the transmitter and receiver, we have developed a $2 \mathrm{D}$ image-based ray tracing simulation tool to calculate the angular information of signal clusters, as shown in Figure 2. The antenna elements are modelled in the ray tracing simulations as vertically polarized omnidirectional antennas. In the ray tracing program, the surfaces of most of the walls of the modelled rooms are assumed to be relatively smooth and all reflecting planes are assumed to be orthogonal. The $2 \mathrm{D}$ ray tracing model is set up using the building structure information for a set of transmitter and receiver positions in a specified indoor environment. The positions of the reference transmitter and receiver elements are taken to approximately coincide with the locations of the actual transmit and receive arrays. Multipath sources are established using the image principle. The ray paths are ordered by the number of the reflections they undergo. The line-of-sight ray is defined as the zeroth order ray and its AOA and AOD are calculated using the geometrical positions of the receiver and transmitter. The rays that impinge on an object and get reflected directly to the receiver are denoted as the first-order rays. The rays from the transmitter which are reflected twice before they reach the receiver are denoted as second-order rays, and so forth. The reflected rays are traced by imaging the transmitter with respect to the walls. Figure 2 shows the image sources

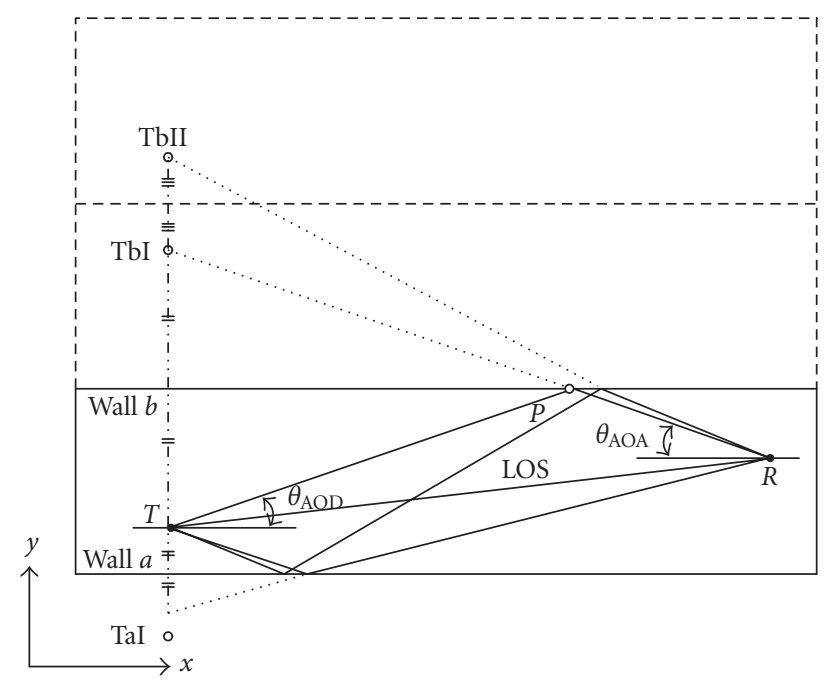

FIGURE 2: A 2D image-based ray tracing model.

due to the walls lying in horizontal plane, where TbI, TbII,... and so forth represent the first, second, and-higher order images of the source. Figure 2 shows one zero-order, two firstorder and one second-order rays in a rectangular room with conducting walls, in which the dotted lines from the images to the receiver denote the virtual paths of the reflected rays.

In this method, both AOD and AOA are traced at the transmitter and receiver sides in the horizontal plane for all reflected rays. For each ray, the AOA is determined from the last reflecting point of the ray before it impinges on the receiver; and the AOD is determined from the first reflecting point after leaving the transmitter. As shown in Figure 2, the AOA and AOD of the first-order ray "TPR" are obtained as

$$
\theta_{\mathrm{AOA}}=\arctan \left(\frac{y_{P}-y_{R}}{x_{R}-x_{P}}\right), \quad \theta_{\mathrm{AOD}}=\arctan \left(\frac{y_{P}-y_{T}}{x_{P}-x_{T}}\right),
$$

where $\theta_{\mathrm{AOA}}$ and $\theta_{\mathrm{AOD}}$ are the AOA and AOD of the ray with respect to the $x$-axis, $\left(x_{T}, y_{T}\right)$ is the position of the transmitter, $\left(x_{R}, y_{R}\right)$ is the position of the receiver, and $\left(x_{P}, y_{P}\right)$ is the position of the reflection point $P$.

The amplitude of the $l$ th ray impinging on the receiver is given by

$$
\beta_{l}=\beta_{0} \sqrt{G_{T}} \sqrt{G_{R}} L\left(d_{l}\right) \prod_{j} \Gamma\left(\xi_{j l}\right) \prod_{k} T\left(\xi_{k l}\right),
$$

where $G_{T}$ and $G_{R}$ are the gains of transmit and receive antennas, respectively, $\beta_{0}$ is the free space electric field at 1 meter away from the transmitter, $L\left(d_{l}\right)$ is the path loss for the $l$ th component having path length $d_{l}, \prod_{j} \Gamma\left(\xi_{j l}\right)$ and $\prod_{k} T\left(\xi_{k l}\right)$ are the total reflection and transmission coefficients, respectively. In grouping multipath rays into clusters according to the similarity of their angular features, we have utilized the same algorithm as in [17] to distinguish the effective clusters 


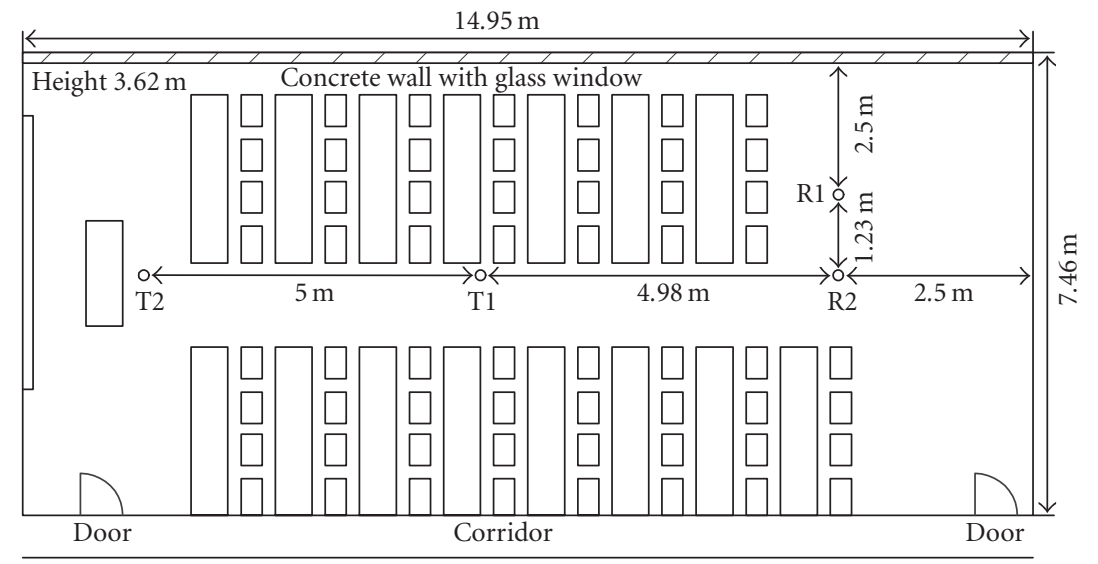

FIgURE 3: A specified classroom environment.

and obtain their mean AOAs and AODs. Once the mean AOAs and AODs of signal clusters are obtained, the rays within each cluster are assumed to follow the Laplacian distribution [17] with certain angle spreads. We will later show how the angle spread influences signal correlation.

\subsection{The MIMO channel matrix}

Although it is possible to use ray tracing to directly calculate the channel transfer function, the computational load even for a simple indoor scenario can be very high. Hence, in the present case, we use ray tracing only for obtaining the numbers and their corresponding angular details of effective signal clusters. Now using the method given in [10], we construct the channel matrix so that the intrinsic correlation features at both ends of the MIMO channel are preserved.

Using the angular detail of signal clusters available at both the transmitter and receiver, we can obtain covariance matrices as

$$
R_{x}=\left[\rho_{i j}\right] \text { for } x=R \text { or } T, i, j=1, \ldots, \operatorname{Mor} P,
$$

where the entries of the matrices are the spatial correlation coefficients between any two antenna elements at either the transmitter or receiver. For NLOS Rayleigh channels, the correlation coefficient between the $i$ th and $j$ th antennas, $\rho_{i j}$ is calculated using (9) and (10). For LOS Ricean channels, $\rho_{i j}$ is calculated using (11) to account for LOS effect.

After the covariance matrices at both sides are obtained, using the Kronecker method, the MIMO channel matrix $H$ with $P$ transmit and $M$ receive antennas can be formed as $[5,10,20]$

$$
H=\left(R_{R}\right)^{1 / 2} G\left[\left(R_{T}\right)^{1 / 2}\right]^{T}
$$

where $G$ is an $M \times P$ matrix with i.i.d. elements, $(\cdot)^{1 / 2}$ represents any matrix square root such that $R^{1 / 2}\left(R^{1 / 2}\right)^{*}=R,(\cdot)^{T}$ denotes matrix transposition, $R_{R}$ and $R_{T}$ are the covariance matrices at the receiver and transmitter sides, respectively.

\section{IMPACT OF SIGNAL CLUSTERING ON INDOOR MIMO PERFORMANCE}

In this section, we use the proposed hybrid MIMO channel model to assess the effect of signal clustering in a typical indoor environment based on simulations and measurements. To use the model, we need information on the physical features of a specified indoor environment so that ray tracing can be utilized to detect the number of signal clusters and calculate their angular details. Using the above information, we can calculate the covariance matrices at both ends using (10), (11), and (14), taking into account the presence or absence of the line-of-sight component. The full MIMO channel matrix is then generated using (15).

First, we validate the proposed model by comparing simulations with our own measurement. The practical indoor environment chosen for validation is a rectangular-shaped classroom located on the 23rd floor of a 28-storey tower building of the University of Technology, Sydney (UTS), as depicted in Figure 3. The classroom has dimensions of $14.95 \times 7.46 \mathrm{~m}^{2}$ with a height of $3.62 \mathrm{~m}$, containing a number of wooden desks and plastic chairs, and is enclosed on one side by a concrete wall incorporating a wide metal-framed glass window. The other three sides of the room have brick internal walls. The room has two entrances which are made of two wooden frames to which are fixed wooden doors that open into a closed concrete-walled corridor. It is assumed that for simulations, an MIMO system with a $P$-element transmit and an $M$-element receive antenna arrays is deployed in the room. Without the loss of generality, the number of transmit and receive array elements are assumed to be the same $(P=M)$ for the MIMO calculations. Both arrays are assumed to have identical elements. The transmit and receive arrays are assumed to lie in the same horizontal plane and the locations of the transmitter as well as the receiver are varied within the horizontal plane. Obviously, in the classroom, a line-of-sight component is always present between the transmit and receive antennas. Applying the image-based ray tracing method to the classroom environment by incorporating the building structure information taken from the blueprint, we have calculated the angular information, that 
TABLE 1: Dielectric properties of modelled building materials (taken from [28]).

\begin{tabular}{lccr}
\hline Material & Relative permittivity & Conductivity $(\mathrm{S} / \mathrm{m})$ & Complex relative permittivity \\
\hline Brick & 4 & 0.003 & $4-j 0.05$ \\
Concrete & 9 & 0.05 & $9-j 0.92$ \\
\hline
\end{tabular}

TABLE 2: AOA and AOD obtained from ray tracing simulations.

\begin{tabular}{cccccccccc}
\hline Tx locations & \multicolumn{3}{c}{ T1 } & & & & T2 & \\
\hline Rx locations & & R1 & & & R2 & & R1 & R2 \\
\hline & AOA & AOD & AOA & AOD & AOA & AOD & AOA & AOD \\
\hline \multirow{3}{*}{ NLOS cluster } & 129 & 51 & 0 & 0 & 148 & 32 & 0 & 0 \\
& 184 & 176 & 124 & 56 & 185 & 175 & 143 & 37 \\
& 353 & 7 & 236 & 304 & 221 & 319 & 217 & -323 \\
\hline LOS & 240 & 300 & - & - & 355 & 187 & 7 & 180 & 0 \\
\hline
\end{tabular}

is, the AOAs and AODs of multipath components. The reflection coefficients for the walls are evaluated using the data given in Table 1 that was gleaned from measurement results presented in [28] for the same building. The simulations were performed for a carrier frequency of $5.2 \mathrm{GHz}$. Using the same algorithm as in [17], we can obtain the mean AOAs and AODs of the respective clusters via a computer program which displays a visual image of the processed data allowing the user to identify clusters by visual examination.

For comparing measurements with simulations made inside the room, two representative scenarios are considered in terms of transmitter and receiver pairs, namely, transmit positions T1, T2 and receive positions $\mathrm{R} 1, \mathrm{R} 2$ as shown in Figure 3. Thus, a total of four reference transmitter-receiver (T-R) pairs, T1R1, T1R2, T2R1, and T2R2, can be formed for making the comparison. Assuming that the transmit array contains $P$ antenna elements and the receive array contains $M$ antenna elements, for each T-R pair, we get a $P \times M$ MIMO connection. Using the ray tracing method, for each T-R pair, the mean AOAs and AODs of effective signal clusters are obtained and tabulated in Table 2.

\subsection{Effect of signal clusters on spatial correlation}

Since the degree of the spatial correlation determines the achievable MIMO capacity gain, we first investigate the effect of clustering propagation as well as the presence of the LOS component on the spatial correlation. Figure 4 shows the plot of the spatial correlation coefficient between received signals at two adjacent antenna elements as a function of array interelement spacing for the four reference T-R pairs. The rays within clusters are assumed to follow Laplacian distribution with an angle spread (AS) of $22^{\circ}$, a value that is gleaned from the published measured data [17] for typical indoor scenarios. The Ricean factor $K$ is assumed to be 2 $(3 \mathrm{~dB})$ in these calculations. The correlation results are compared with those for the unclustered case calculated using (6) [33]. Figure 4 reveals that the unclustered case has lower values of correlation coefficients. In Figure 5 a the spatial correlation coefficient is plotted as a function of the angle spread of clusters for the T-R pair T1R1 with a Ricean factor $K=2$.

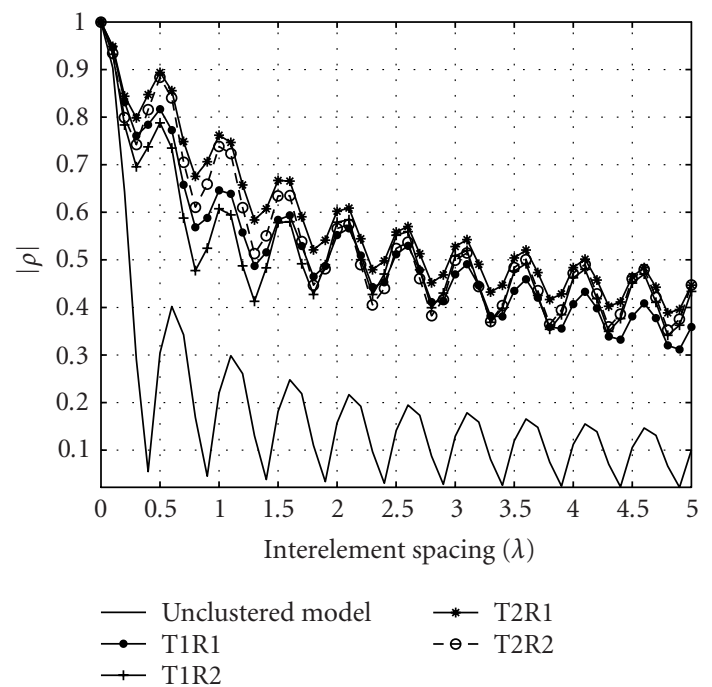

FIGURE 4: Correlation coefficients between two antenna elements.

Figure $5 \mathrm{~b}$ provides results to compare the impact of the angle spread of clusters with and without LOS condition. Here the NLOS results were obtained by artificially blocking the LOS ray for the same T1R1 pair. It is clear from the plot that the effect of the angle spread of clusters is more significant for NLOS scenarios as compared to LOS scenarios. A large differential can be seen between the results of the NLOS and LOS scenarios when the angle spread varies between $5^{\circ}-45^{\circ}$. This can be attributed to the absence or presence of the dominant LOS component. Without the dominant LOS component, the correlation becomes smaller. This makes the investigation of the effect of Ricean $K$ factor on the spatial correlation for LOS channels very appropriate. The spatial correlation between two antenna elements as a function of $K$ factor for the T-R pair T1R 1 for an angle spread of $22^{\circ}$ is presented in Figure 6. It is clear from the figure that the Ricean $K$ factor has a significant impact on the signal correlation. The correlation coefficient increases rapidly when $K$ increases from 0.2 to 6 . This result confirms that, for an indoor MIMO system operating in a Ricean channel, the presence of the LOS 


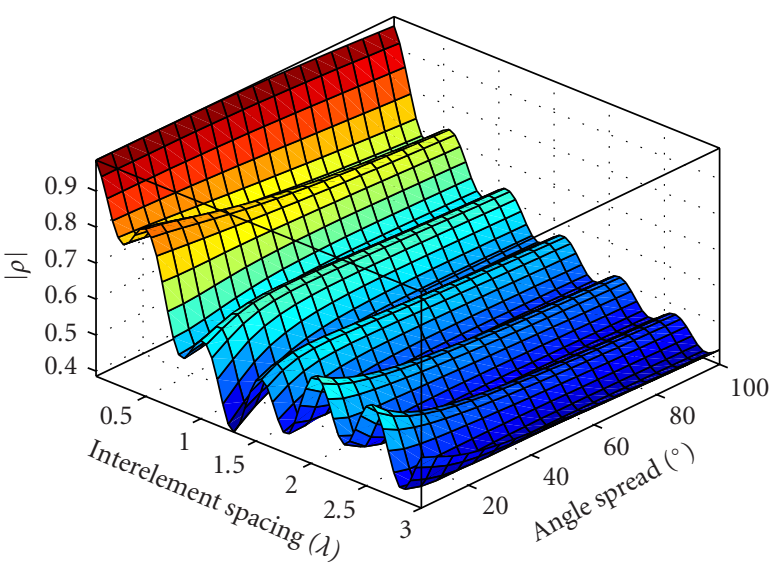

(a)

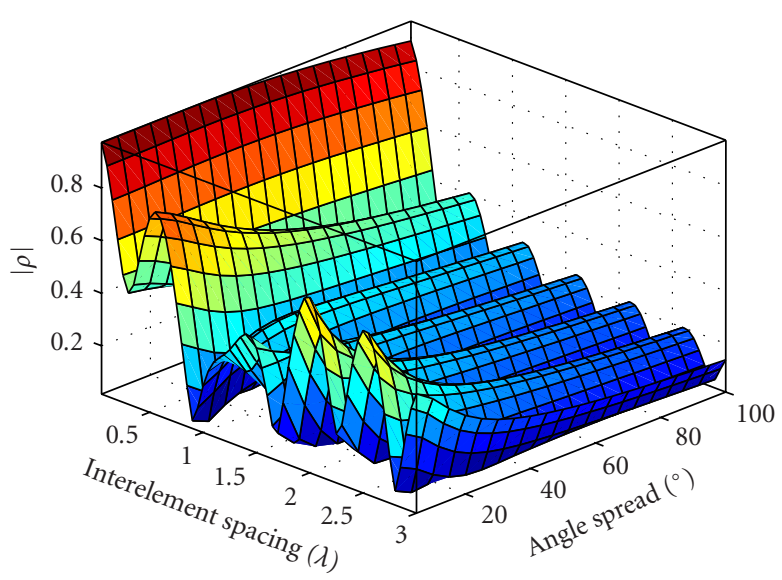

(b)

FIGURE 5: Effect of the angle spread on spatial correlation. (a) Correlation coefficient as a function of angle spread of clusters for LOS channel. (b) Correlation coefficient as a function of angle spread of clusters when LOS is blocked.

component can cause an increase in correlation which can be an impediment for achieving higher capacity.

\subsection{Measurements and comparisons}

Here we attempt to validate our channel model with measurement data collected in the same classroom as in Figure 3 in terms of achievable MIMO capacity. In addition, we will show a comparison with the unclustered case to highlight the effect of signal clustering in indoor environments. The measurements were performed using a vector network analyzer (VNA) HP 8720A at a centre frequency of $2.45 \mathrm{GHz}$ for vertical polarization inside the same classroom as shown in Figure 3. Both the transmit and receive arrays were formed as synthetic arrays using commercially available sleeve dipole antennas in order to avoid mutual coupling and also to reduce the complexity and cost of the MIMO measurement. The measurements were conducted during weekends to avoid the movement of people so as to approximate a quasistatic channel condition. To obtain a virtual transmit array, a computer-controlled angular scanner moved a sleeve

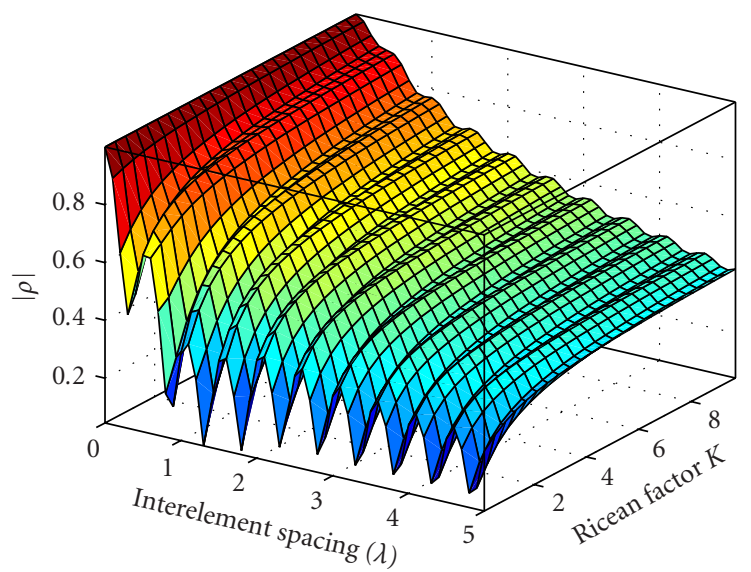

FIGURE 6: Correlation coefficient for LOS channel as a function of Ricean factor $K$.

dipole antenna around a circle to form a virtual four-element uniform circular array (UCA) with a radius of half a wavelength. At the receiver side, a virtual receive array was obtained using a computer-controlled X-Y scanning system. A synthetic uniform rectangular array was formed by moving a dipole antenna over the horizontal plane. For each transmit and receive pair, 801 frequency response measurements were acquired within a bandwidth of $120 \mathrm{MHz}$. In the measurements, the transmit antenna was located at position T2 as indicated in Figure 3, whilst the receiver was moved between different positions. During all the measurements, the heights of both transmit and receive antennas were fixed at $1.7 \mathrm{~m}$ above the floor level.

A plot of the cumulative distribution function (CDF) of the MIMO capacity obtained using both simulation and measurement for the reference T-R pairs T2R1 and T2R2 is shown in Figures $7 \mathrm{a}$ and $7 \mathrm{~b}$, respectively. The MIMO connections were established between a virtual four-element uniform circular transmit array with a radius of half a wavelength and a virtual four-element uniform linear receive array with half a wavelength interelement spacing at a centre frequency of $2.45 \mathrm{GHz}$. With reference to Figure 3, the receive uniform linear array (ULA) was placed to lie parallel to the concrete wall and the corridor. For each receive position, the measured $4 \times 4$ MIMO channel transfer matrices were normalized to calculate the experimental channel capacity using (2) under an equal power allocation scheme with an average SNR $=20 \mathrm{~dB}$. Later, using ray tracing simulations, the number of effective clusters and their AODs and AOAs were calculated for the same scenarios as in Figure 3 at a centre frequency of $2.45 \mathrm{GHz}$. Using the Laplacian distribution with an angle spread of $22^{\circ}$ to represent the rays within each cluster, the correlation matrices at the transmitter and receiver were obtained via (9), (10), and (11), and then 15000 random MIMO channel matrix realizations were simulated using (15). We have assumed in the simulations the same transmit and receive antenna array configuration as used in the measurements. The Ricean $K$ factors for simulations were taken directly from experimental values by applying the moment-method [35] on the measured data. 
Thus, $K=3.5$ was obtained for the reference T-R pair T2R1 and $K=3.6$ for the reference T-R pair T2R2. The average receive SNR was set to $20 \mathrm{~dB}$ in both the experiment and simulations. The results in Figures $7 \mathrm{a}$ and $7 \mathrm{~b}$ show that there is a good agreement between the simulated and measured results for the two reference T-R pairs considered for comparison. While there is an agreement on both trend and median value of capacity, a discrepancy that is seen either for the very high capacity or very low capacity may be due to the inadequate number of measurement data $(801 \times 4=3204$ MIMO measured realizations for each link) as compared to 15000 realizations for the simulation. The simulation for unclustered case assumes all NLOS multipath components, except the LOS component, to uniformly span the range of $[0,2 \pi)$. The correlation due to NLOS unclustered multipath components was calculated using (6). As shown, the unclustered model overestimates the capacity by $2 \mathrm{bits} / \mathrm{s} / \mathrm{Hz}$ than that obtained by both the measurement and the simulation using the cluster model. The channel capacity calculated for the four reference T-R pairs with $K=3.5$, angle spread of $22^{\circ}$ as compared with i.i.d. scenarios fixing the SNR at $20 \mathrm{~dB}$ for a centre frequency of $2.45 \mathrm{GHz}$ is shown in Figure 7c. The comparison with i.i.d. case is included here since the capacity of the i.i.d. uncorrelated channels can be considered as the upperbound on the achievable MIMO capacity. The $4 \times 4$ MIMO connections for the four reference T- $\mathrm{R}$ pairs are assumed to be established between a four-element uniform circular array having a radius of half a wavelength at the transmitter and a four-element uniform linear array with half a wavelength interelement spacing at the receiver. Although, as seen in Figure $7 c$, the results for all four reference T-R pairs show similar trends, due to the presence of LOS component, the capacities obtained are smaller than that of the i.i.d. channels. The relatively higher values of capacity obtained for the pair T1R1 could be due to the effect of angular orientation of the ULA which will be investigated in the next section.

\subsection{Effect of array topologies in clustering indoor environments}

As we have seen in previous section, the clustering of multipath signals affects the signal correlation between array elements which in turn has bearing on the achievable MIMO capacity. Due to the use of multiple-element antennas to exploit multipath fading, the correlation between antenna elements can be sensitive to the array topology and subsequent changes in the angular characteristics of multipath waves $[5,9,36]$. As the propagation of multipath waves is random, even within the same physical indoor environment, for a given link pair, with, say, transmitter end fixed, the propagation characteristics such as AOA, AOD, and so forth, can vary with the change at the receive end. Thus, the choice of array topology to exploit the signal clustering in indoor environments assumes practical significance for indoor MIMO applications.

To gain insights on these issues, we investigate and compare the performances of five- and six-element antenna arrays operating in a specified indoor environment. The considered arrays are arranged in different topologies as shown

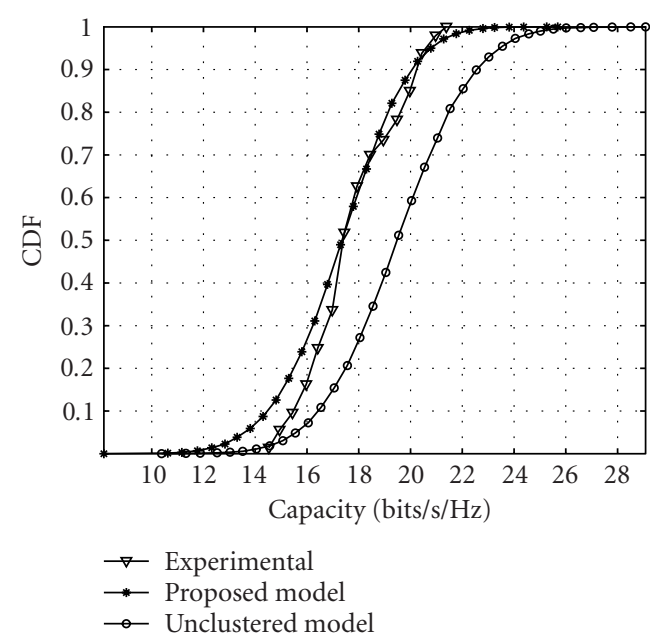

(a)

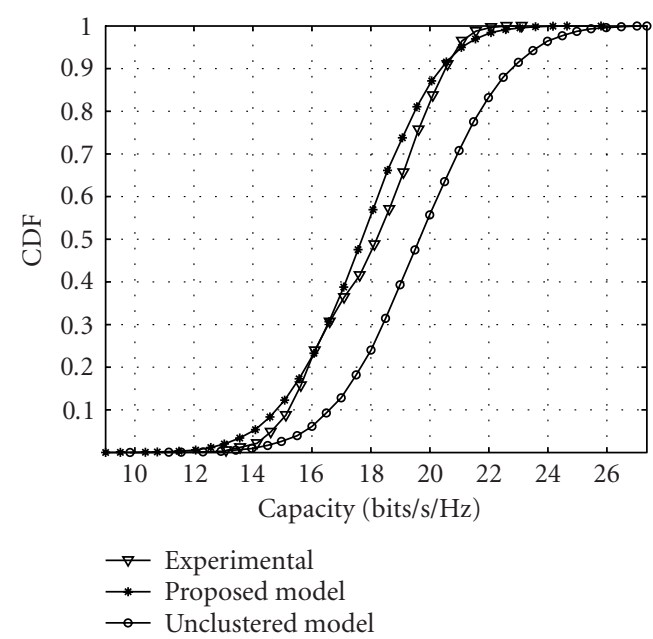

(b)

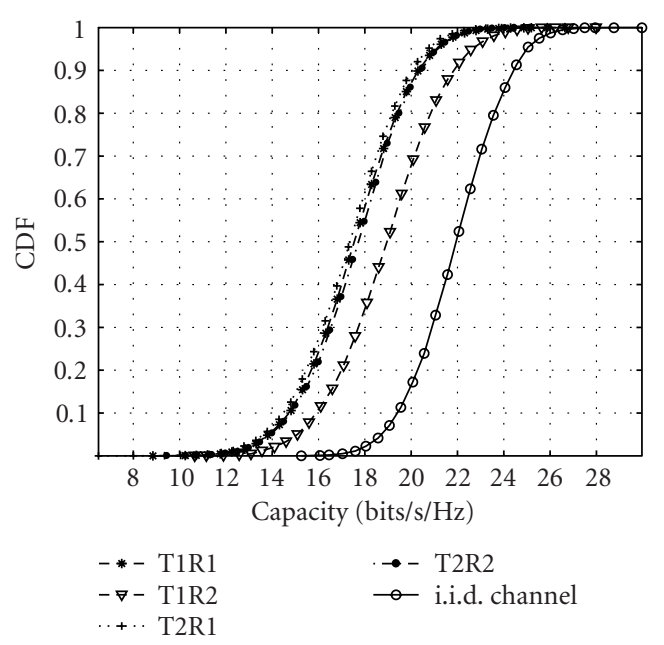

(c)

Figure 7: Comparison of capacity for $4 \times 4$ MIMO channels. (a) Capacity comparison for T-R pair T2R1 (angle spread $=22^{\circ}, K=$ 3.5). (b) Capacity comparison for T-R pair T2R2 (angle spread $=$ $22^{\circ}, K=3.6$ ). (c) Simulated capacity comparison for four T-R pairs versus i.i.d. channels. (a), (b), and (c) $\mathrm{SNR}=20 \mathrm{db}$. 


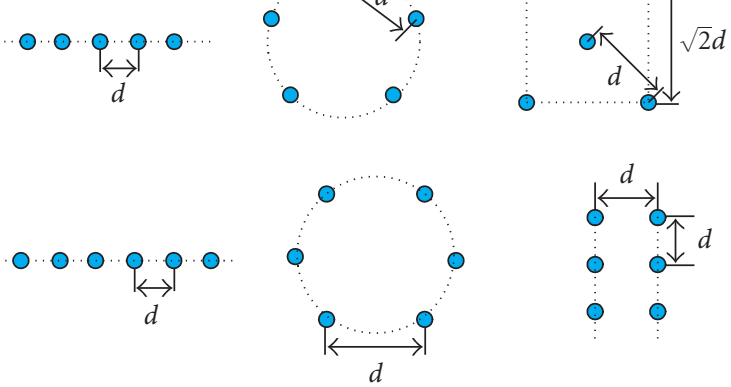

FIGURE 8: Antenna array topologies considered.

in Figure 8. The five-element array topologies considered include a ULA, a UCA with a radius of $0.85 d$, and a modified square array (MSA) in which four elements are arranged as a square planar array with the fifth element located at its centre. The six-element array topologies considered include a ULA, a UCA with a radius of $d$, and a $2 \times 3$ planar uniform rectangular array to be denoted as URA. The interelement spacing " $d$ " is assumed to be half a wavelength. Our aim here is to find out an array topology that performs better in indoor clustering environments, from the point of view of achievable MIMO capacity.

Here we keep a uniform linear array at the transmit side fixed to maintain the same fading correlation condition, and at the receive side, different array topologies as shown in Figure 8 are considered, thereby obtaining either a $5 \times 5$ or $6 \times 6$ MIMO connection. The receive arrays are rotated to simulate various orientations of the receiver. The mean capacity is calculated when the receive array is rotated in the horizontal plane by an angle of $3^{\circ}$ with respect to array centre. The variance of the channel capacity can be used as a parameter that measures the angle sensitivity of the receive array topology. Since the variance of the capacity is due to the rotation of the array, we would like to denote this variance as the angular capacity variation (ACV) of a given array. For an MIMO system employing different array topologies in the same propagation scenario, a smaller value for the ACV parameter reflects lower sensitivity with respect to the angular characteristics of signal clusters. Tables 3 and 4 provide the calculated data on ACV, mean, maximum and minimum capacities for ULA, UCA, MSA, URA topologies for the four reference T-R pairs inside the same classroom as in Figure 3. In addition, results for an i.i.d. case are also included for the sake of comparison. For the i.i.d. case, no angle effect is considered and hence ACV values are always less than 0.1 .

All simulations use the same parameters: $K=3.5$, angle spread $=22^{\circ}$, and an average SNR of $20 \mathrm{~dB}$ at a centre frequency of $5.2 \mathrm{GHz}$. A comparison of the mean MIMO capacity for $5 \times 5$ and $6 \times 6$ MIMO for only one reference T-R pair T2R1 is shown in Figure 9 which demonstrates the effects of array topology. It must be mentioned that the shapes of the plots for the other reference T-R pairs appear similar to Figure 9 and hence are not repeated here. The capacity parameters obtained for an i.i.d. channel are also included in the same figure for comparison.

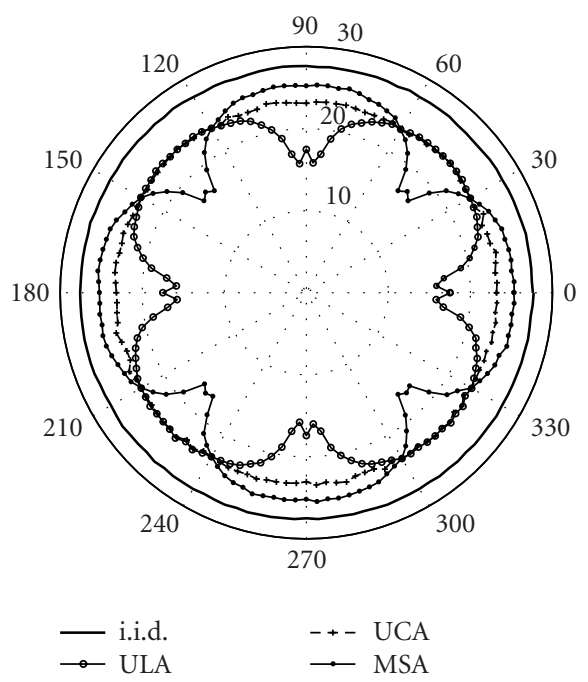

(a)

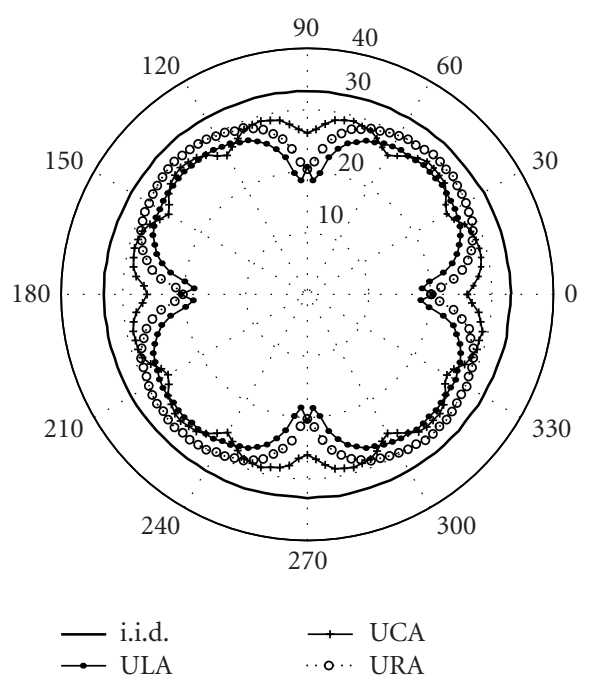

(b)

FIGURE 9: Angular performance for different receive array topologies. (a) $5 \times 5$ MIMO for T-R pair T2R1. (b) $6 \times 6$ MIMO for T-R pair T2R1.

The results given in Tables 3 and 4 indicate that amongst all the topologies considered, the uniform circular array performs best, that is, UCA has the smallest angular capacity variations and the largest values of mean capacity within each group. However, differences in results for five- and sixelement arrays are also observed. Among the five-element arrays, the MSA, for all the scenarios considered, has achieved the highest maximum capacity with a mean capacity similar to the corresponding UCA. However, the ACV obtained for MSA is also the largest of the three arrays considered. This is evident from Figure 9a for T-R pair T2R1. For six-element arrays, for the reference T-R pairs T1R1 and T1R2, the largest angular capacity variation equal to 2.92 for T1R1 and 3.05 for T1R2 is obtained for the URA as tabulated in Table 4. However, for the T-R pairs T2R1 and T2R2, the largest variations of angular capacity equal to 3.23 and 3.154 are obtained for the ULA. For both $5 \times 5$ and $6 \times 6$ MIMO cases, the 
TABLE 3: Angle sensitive parameters for different array topologies (T-R pairs T2R1 and T2R2).

\begin{tabular}{|c|c|c|c|c|c|c|c|c|c|}
\hline \multirow{2}{*}{$\mathrm{T} 2$} & \multicolumn{5}{|c|}{$5 \times 5$} & \multicolumn{4}{|c|}{$6 \times 6$} \\
\hline & & ULA & UCA & MSA & i.i.d. & ULA & UCA & URA & i.i.d. \\
\hline \multirow{4}{*}{ R1 } & $\mathrm{ACV}$ & 2.58 & 0.29 & 2.71 & 0.06 & 3.23 & 1.01 & 2.97 & 0.07 \\
\hline & Mean & 21.17 & 23.11 & 23.01 & 27.59 & 25.02 & 27.75 & 27.58 & 33.04 \\
\hline & $\operatorname{Max}$ & 23.53 & 24.35 & 25.34 & 27.71 & 28.08 & 28.93 & 30.32 & 33.21 \\
\hline & Min & 15.25 & 22.64 & 16.35 & 27.44 & 17.73 & 25.78 & 19.94 & 32.9 \\
\hline \multirow{4}{*}{ R2 } & $\mathrm{ACV}$ & 2.51 & 0.18 & 2.72 & - & 3.15 & 1.02 & 2.99 & - \\
\hline & Mean & 21.36 & 23.27 & 23.21 & - & 25.43 & 27.96 & 27.75 & - \\
\hline & Max & 23.83 & 23.67 & 25.52 & - & 28.34 & 29.32 & 30.64 & - \\
\hline & Min & 15.76 & 22.84 & 16.66 & - & 18.45 & 26.04 & 20.19 & - \\
\hline
\end{tabular}

TABLE 4: Angle sensitive parameters for different array topologies (T-R pairs T1R1 and T1R2).

\begin{tabular}{|c|c|c|c|c|c|c|c|c|c|}
\hline \multirow{2}{*}{$\mathrm{T} 1$} & \multicolumn{5}{|c|}{$5 \times 5$} & \multicolumn{4}{|c|}{$6 \times 6$} \\
\hline & & ULA & UCA & MSA & i.i.d. & ULA & UCA & URA & i.i.d. \\
\hline \multirow{4}{*}{$\mathrm{R} 1$} & $\mathrm{ACV}$ & 2.16 & 0.32 & 2.58 & 0.06 & 2.71 & 0.92 & 2.92 & 0.07 \\
\hline & Mean & 22.23 & 23.81 & 24.24 & 27.59 & 26.53 & 29.3 & 29.24 & 33.04 \\
\hline & Max & 24.63 & 25.11 & 26.58 & 27.71 & 29.36 & 30.51 & 32.1 & 33.21 \\
\hline & Min & 18.31 & 22.94 & 18.44 & 27.44 & 21.7 & 27.84 & 22.45 & 32.9 \\
\hline \multirow{4}{*}{ R2 } & $\mathrm{ACV}$ & 1.94 & 0.18 & 2.48 & - & 2.43 & 1.06 & 3.05 & - \\
\hline & Mean & 21.83 & 23.17 & 23.64 & - & 26.01 & 28.02 & 27.99 & - \\
\hline & $\operatorname{Max}$ & 23.9 & 23.65 & 25.85 & - & 28.57 & 29.29 & 30.99 & - \\
\hline & Min & 18.22 & 22.73 & 18.07 & - & 21.56 & 26.22 & 20.74 & - \\
\hline
\end{tabular}

propagation of signal clusters appears to degrade the achievable capacity. All the above results favourably point towards the uniform circular array, among all the topologies considered, as the possible choice for use in clustering indoor environments.

\subsection{Efficiency of the clustering indoor channels}

In the design and development of indoor MIMO systems, the optimization of the performance-to-cost ratio requires finding an optimum number of antenna elements for a specified indoor channel which can best exploit the intrinsic diversity. This topic has drawn much attention in terms of antenna selections for the MIMO technique $[14,15]$. The objective of the antenna selection is to achieve the best performance-tocost ratio so as to obtain the optimized capacity and reduce the expensive RF chains. As the achievable MIMO capacity is heavily reliant on the multipath fading channels, the antenna selection criteria for indoor MIMO systems must account for the characteristics of clustering propagation. In this section, rather than concentrating on antenna selection schemes, we wish to focus on the channel efficiency of indoor MIMO systems in a specified clustering indoor environment in order to gain insights that may aid in the design of antenna selection criteria. The channel efficiency here refers to the achievable subchannel capacity per used antenna, denoted in terms of bits/s/Hz/element.

A finite number of incident clusters allows a particular correlated channel to accommodate only a finite number of spatial subchannels with sufficient subchannel gain for

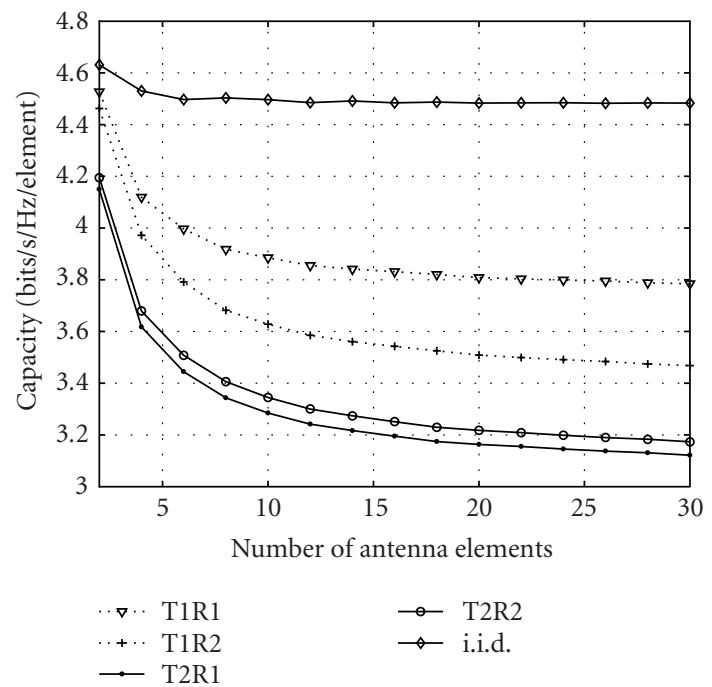

Figure 10: Subchannel efficiency when ULAs are used at both the transmitter and receiver.

transmitting the signals. Therefore, the efficiency per element will decrease when the number of elements of the antenna array increases. This is evident from the simulations shown in Figure 10 for the four reference T-R pairs: T1R1, T1R2, T2R1, and T2R2 inside the classroom as shown in Figure 3. In all the cases considered in this section, uniform linear arrays with interelement spacing of half a wavelength are used 


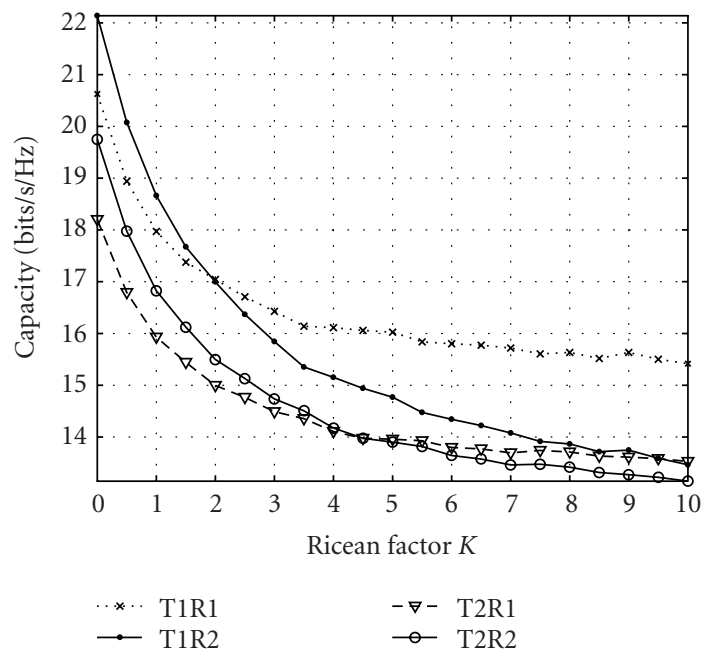

FIgURE 11: Effect of the $K$ factor in clustering channels.

at both the transmitter and receiver. Both the arrays are assumed to be located inside the classroom parallel to the corridor and have the same number of elements. We restrict our attention only for ULA here, as our aim is to introduce the idea of channel efficiency for clustered indoor channels. The simulation parameters are Ricean factor $K=3.5$, angle spread is $22^{\circ}$, and the average SNR is $20 \mathrm{~dB}$ at a centre frequency of $5.2 \mathrm{GHz}$. For the sake of comparison, the channel efficiency for an ideal i.i.d. channel is also included in Figure 10, since the efficiency for i.i.d. channels can be considered to be an upperbound. For all the pairs except for the i.i.d. case, the efficiency per antenna element decreases with the increase in the number of array elements. The subchannel efficiency for i.i.d. channels is almost a constant, but for the cluster case, the subchannel efficiency for all the four reference $\mathrm{T}-\mathrm{R}$ pairs decreases by $18 \%-25 \%$ when the number of elements of ULA increases from 2-20. The subchannel efficiency has a decreasing trend and the differences seen in the results for the same indoor environment can be attributed to the differing angular properties of clusters due to the different receiver and transmitter locations. These results emphasize that the antenna selection process for indoor MIMO systems must incorporate the effect of propagating clusters and their angular features.

\subsection{Effect of Ricean factor in LOS clustering channels}

We have already demonstrated the effect of Ricean factor $K$ on the spatial correlation of received signals in the previous section. Here, we investigate its effect on MIMO performance. The effect of the Ricean factor on the equal power capacity for the four reference T-R pairs is plotted in Figure 11. The simulation results in Figure 11 are based on $4 \times 4$ MIMO connections with ULAs placed parallel to the corridor at each end inside the classroom as shown in Figure 3. The interelement spacing for the ULAs is half a wavelength. All simulations use the following parameters: angle spread is $22^{\circ}$ and the SNR is $20 \mathrm{~dB}$ at a centre frequency of $5.2 \mathrm{GHz}$. The

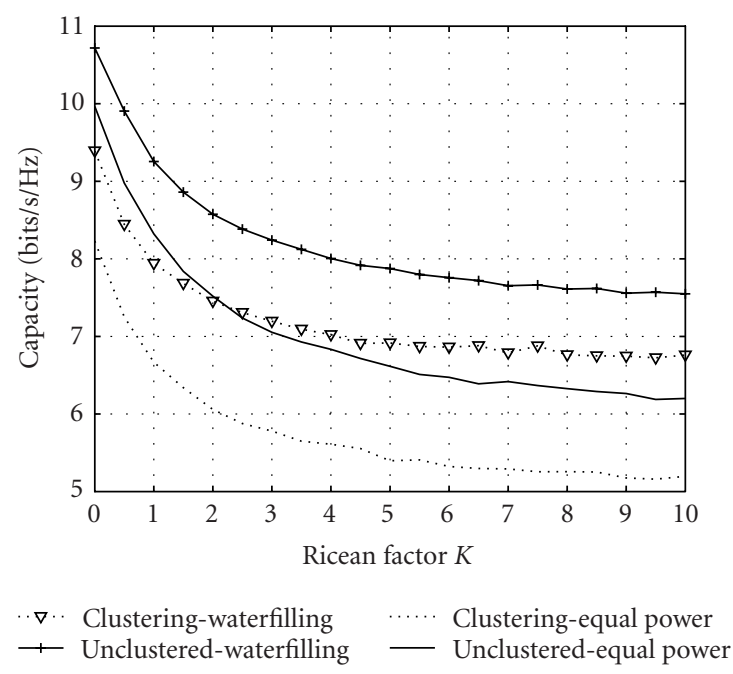

Figure 12: Comparison of the effect of $K$ factor in clustering and unclustered scenarios for T2R1.

capacity shows a decreasing trend with increasing values of the $K$ factor and is consistent for all the four T-R pairs. Due to differing angular characteristics of clusters and angular sensitivities of ULAs, the effect of the $K$ factor varies for different links. For the communication pairs T1R2 and T2R2 for which the LOS component is parallel to the corridor as well as to the axis of ULA, the effect is more significant than the other two T-R links. Further, the effect of the Ricean factor on the two power allocation schemes (equal power and waterfilling) in both clustering and unclustered scenarios are compared. For the all four T-R pairs, similar trends can be seen to occur. Figure 12 shows the comparison of the two MIMO power allocation schemes for the T-R pair T2R1 in both clustering and unclustered scenarios as a function of the Ricean factor $K$ with the following fixed parameters: angle spread is $22^{\circ}$ for clustering scenarios and the SNR is $10 \mathrm{~dB}$ at a centre frequency of $5.2 \mathrm{GHz}$. The $4 \times 4 \mathrm{MIMO}$ uses ULAs with half a wavelength interelement spacing at both ends. The equal power and waterfilling capacities were respectively obtained using (4) and (5). The correlation caused by unclustered NLOS components is obtained using (6) assuming NLOS rays to be uniformly distributed over $[0,2 \pi)$. As can be seen, in all the cases, the obtainable capacity decreases with increasing values of the $K$ factor. For the equal power allocation scheme, the effect of Ricean factor $K$ on both clustering and unclustered scenarios is similar. However, for waterfilling power allocation scheme, some differences are observed between clustering and unclustered scenarios. When the value of $K$ factor increases from $0-6$, the waterfilling and equal power capacities for unclustered scenario decrease by about $28.7 \%$ and $34.3 \%$; and for the clustering scenario, both capacities decrease by about $26.5 \%$ and $34.1 \%$, respectively. For both clustering and unclustered scenarios, the divergence between equal power and waterfilling capacities increase with the increase in the value of the $K \mathrm{fac}-$ tor. This is justified by the waterfilling theory where more power is allocated to the best subchannel. 
From the above discussion, we conclude that the effect of the Ricean factor $K$ is negative for the two MIMO power allocation schemes, namely, equal power and waterfilling algorithms, in both clustering and unclustered LOS scenarios.

\section{CONCLUSIONS}

In this paper, we have proposed a hybrid MIMO channel model for indoor propagation environments, which is utilized to investigate the effect of signal clusters on the performance of indoor MIMO systems. Our studies reveal that the clustering of multipath waves affects the MIMO channel capacity by influencing the spatial correlation among MIMO subchannels. Due to the clustering of multipath waves, the achievable MIMO capacity in indoor environments was found to be always lower than that of the ideal i.i.d. scenarios. Comparisons with measurement results also indicate that the unclustered uniform model will overestimate the actual MIMO performance. The simulations in a specified indoor environment show that the impact of the angle spread of clusters on spatial correlation is more significant for Rayleigh channels than for Ricean channels. In Ricean clustering channels, an increase in the value of the Ricean factor degrades MIMO capacity since the dominance of the LOS component tends to increase the spatial correlation. The investigations on angle sensitivity for five- and six-element array topologies indicate that uniform circular arrays have the best angular performance and achieve the highest mean capacity in clustering indoor channels when compared with other types of array topologies. The subchannel efficiency in clustering indoor environments is also investigated to gain insights for the design of antenna selection criteria for indoor MIMO systems. The results predicted using the proposed hybrid model compare well with measurement results thus emphasizing its usefulness as an efficient and accurate means to evaluate practical indoor MIMO performance.

\section{ACKNOWLEDGMENT}

The project is funded by the Australian Research Council through a linkage grant program with Singtel Optus Pty Limited as the industry partner.

\section{REFERENCES}

[1] D. Gesbert, M. Shafi, D.-S. Shiu, P. J. Smith, and A. Naguib, "From theory to practice: an overview of MIMO spacetime coded wireless systems," IEEE J. Select. Areas Commun., vol. 21, no. 3, pp. 281-302, 2003.

[2] A. J. Paulraj, D. A. Gore, R. U. Nabar, and H. Bölcskei, "An overview of MIMO communications-A key to Gigabit Wireless," Proc. IEEE, vol. 92, no. 2, pp. 198-218, 2004.

[3] G. J. Foschini and M. J. Gans, "On limits of wireless communications in a fading environment when using multiple antennas," Wireless Personal Communications, vol. 6, no. 3, pp. 311335, 1998.

[4] I. E. Telatar, "Capacity of multi-antenna Gaussian channels," European Transactions on Telecommunications, vol. 10, no. 6, pp. 585-595, 1999.
[5] D.-S. Shiu, G. J. Foschini, M. J. Gans, and J. M. Kahn, "Fading correlation and its effect on the capacity of multielement antenna systems," IEEE Trans. Commun., vol. 48, no. 3, pp. 502513,2000

[6] J. P. Kermoal, L. Schumacher, K. I. Pedersen, P. E. Mogensen, and F. Frederiksen, "A stochastic MIMO radio channel model with experimental validation," IEEE J. Select. Areas Commun., vol. 20, no. 6, pp. 1211-1226, 2002.

[7] P. Kyritsi and D. C. Cox, "Correlation properties of MIMO radio channels for indoor scenarios," in Proc. 35th Asilomar Conference on Signals, Systems and Computers (ACSSC '01), vol. 2, pp. 994-998, Pacific Grove, Calif, USA, November 2001.

[8] M. T. Ivrlac, W. Utschick, and J. A. Nossek, "Fading correlations in wireless MIMO communication systems," IEEE J. Select. Areas Commun., vol. 21, no. 5, pp. 819-828, 2003.

[9] P. Kyritsi, D. C. Cox, R. A. Valenzuela, and P. W. Wolniansky, "Effect of antenna polarization on the capacity of a multiple element system in an indoor environment," IEEE J. Select. Areas Commun., vol. 20, no. 6, pp. 1227-1239, 2002.

[10] D. Chizhik, F. Rashid-Farrokhi, J. Ling, and A. Lozano, "Effect of antenna separation on the capacity of BLAST in correlated channels," IEEE Commun. Lett., vol. 4, no. 11, pp. 337-339, 2000.

[11] D. Hampicke, M. Landmann, C. Schneider, et al., "MIMO capacities for different antenna array structures based on double directional wide-band channel measurements," in Proc. IEEE 56th Vehicular Technology Conference (VTC '02), vol. 1, pp. 180-184, Vancouver, British Columbia, Canada, September 2002.

[12] A. Abdi and M. Kaveh, "A space-time correlation model for multielement antenna systems in mobile fading channels," IEEE J. Select. Areas Commun., vol. 20, no. 3, pp. 550-560, 2002.

[13] P. F. Driessen and G. J. Foschini, "On the capacity formula for multiple input-multiple output wireless channels: a geometric interpretation," IEEE Trans. Commun., vol. 47, no. 2, pp. 173176, 1999.

[14] A. F. Molisch, "MIMO systems with antenna selection-an overview," in Proc. Radio and Wireless Conference (RAWCON '03), pp. 167-170, Boston, Mass, USA, August 2003.

[15] A. Gorokhov, D. A. Gore, and A. J. Paulraj, "Receive antenna selection for MIMO spatial multiplexing: theory and algorithms," IEEE Trans. Signal Processing, vol. 51, no. 11, pp. 2796-2807, 2003.

[16] A. A. M. Saleh and R. A. Valenzuela, "A statistical model for indoor multipath propagation," IEEE J. Select. Areas Commun., vol. 5, no. 2, pp. 128-137, 1987.

[17] Q. H. Spencer, B. D. Jeffs, M. A. Jensen, and A. L. Swindlehurst, "Modeling the statistical time and angle of arrival characteristics of an indoor multipath channel," IEEE J. Select. Areas Commun., vol. 18, no. 3, pp. 347-360, 2000.

[18] J.-H. Jo, M. A. Ingram, and N. Jayant, "Angle clustering in indoor space-time channels based on ray tracing," in Proc. IEEE 54th Vehicular Technology Conference (VTC '01), vol. 4, pp. 2067-2071, Atlantic City, NJ, USA, October 2001.

[19] C.-C. Chong, C.-M. Tan, D. I. Laurenson, S. McLaughlin, M. A. Beach, and A. R. Nix, "A new statistical wideband spatiotemporal channel model for 5-GHz band WLAN systems," IEEE J. Select. Areas Commun., vol. 21, no. 2, pp. 139-150, 2003.

[20] B. S. Khatri, "Effect on capacity of clustering in indoor MIMO channels," in Proc. IEE Seminar on MIMO: Communications Systems from Concept to Implementations, pp. 15/1-15/7, London, UK, December 2001, Ref. No. 2001/175. 
[21] K.-H. Li, M. A. Ingram, and A. Van Nguyen, "Impact of clustering in statistical indoor propagation models on link capacity," IEEE Trans. Commun., vol. 50, no. 4, pp. 521-523, 2002.

[22] K. Yu, M. Bengtsson, B. Ottersten, D. McNamara, P. Karlsson, and M. A. Beach, "Second order statistics of NLOS indoor MIMO channels based on $5.2 \mathrm{GHz}$ measurements," in Proc. IEEE Global Telecommunications Conference (GLOBECOM '01), vol. 1, pp. 156-160, San Antonio, Tex, USA, November 2001

[23] J. W. Wallace and M. A. Jensen, "Modeling the indoor MIMO wireless channel," IEEE Trans. Antennas Propagat., vol. 50, no. 5, pp. 591-599, 2002.

[24] A. Burr, "Evaluation of capacity of indoor wireless MIMO channel using ray tracing," in Proc. International Zurich Seminar on Broadband Communications, Access, Transmission, Networking (IZS '02), pp. 28-1-28-6, Zurich, Switzerland, February 2002.

[25] H. Hashemi, "The indoor radio propagation channel," Proc. IEEE, vol. 81, no. 7, pp. 943-968, 1993.

[26] H. Nikookar and H. Hashemi, "Phase modeling of indoor radio propagation channels," IEEE Trans. Veh. Technol., vol. 49, no. 2, pp. 594-606, 2000.

[27] M. Lu, T. Lo, and J. Litva, "A physical spatio-temporal model of multipath propagation channels," in Proc. IEEE 47th Vehicular Technology Conference (VTC '97), vol. 2, pp. 810-814, Phoenix, Ariz, USA, May 1997.

[28] H. Suzuki and A. S. Mohan, "Measurement and prediction of high spatial resolution indoor radio channel characteristic map," IEEE Trans. Veh. Technol., vol. 49, no. 4, pp. 1321-1333, 2000.

[29] J.-G. Wang, A. S. Mohan, and T. A. Aubrey, "Analysis of AOA of multipath signals inside buildings: A comparison between MUSIC and Ray Tracing," in Proc. International Symposium on Antennas and Propagation (IASP '96), vol. 1, pp. 189-192, Chiba, Japan, September 1996.

[30] S. Y. Seidel and T. S. Rappaport, "Site-specific propagation prediction for wireless in-building personal communication system design," IEEE Trans. Veh. Technol., vol. 43, no. 4, pp. 879-891, 1994.

[31] G. German, Q. H. Spencer, A. L. Swindlehurst, and R. A. Valenzuela, "Wireless indoor channel modeling: statistical agreement of ray tracing simulations and channel sounding measurements," in Proc. IEEE Int. Conf. Acoustics, Speech, Signal Processing (ICASSP '01), vol. 4, pp. 2501-2504, Salt Lake City, Utah, USA, May 2001.

[32] R. Vaughan and J. B. Andersen, Channels, Propagation and Antennas for Mobile Communications, Institution of Electrical Engineers, London, UK, 2003.

[33] W. C. Jakes, Microwave Mobile Communications, IEEE Press, Piscataway, NJ, USA, 1994.

[34] H. Xu, M. J. Gans, D. Chizhik, J. Ling, P. W. Wolniansky, and R. A. Valenzuela, "Spatial and temporal variations of MIMO channels and impacts on capacity," in Proc. IEEE International Conference on Communications (ICC '02), vol. 1, pp. 262-266, New York, NY, USA, April-May 2002.

[35] L. J. Greenstein, D. G. Michelson, and V. Erceg, "Momentmethod estimation of the Ricean K-factor," IEEE Commun. Lett., vol. 3, no. 6, pp. 175-176, 1999.

[36] A. F. Molisch, M. Steinbauer, M. Toeltsch, E. Bonek, and R. S. Thoma, "Capacity of MIMO systems based on measured wireless channels," IEEE J. Select. Areas Commun., vol. 20, no. 3, pp. 561-569, 2002.
Zhongwei Tang received the M.E. degree in information and telecommunication engineering from the Southeast University (SEU), China, in 1999. He is currently with the Microwave and Wireless Technology Research Laboratory (MWTRL), Information and Communication Group, Faculty of Engineering, University of Technology, Sydney (UTS), Australia, where he has been working towards his Ph.D. degree since 2002.

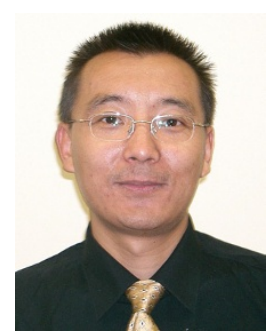
His current research interests include indoor propagation, MIMO channel measurements, characterization and modelling, smart antennas, MIMO systems, and array signal processing.

Ananda Sanagavarapu Mohan is currently a member of the Faculty of Engineering, University of Technology, Sydney (UTS), Australia, where he leads research on antennas, microwaves, wave propagation, and wireless technology. He received a Ph.D. degree in electrical communication engineering from the Indian Institute of Technology, Kharagpur, India, and was a Scientist and Senior Scientist at the Research and Training Unit for Navigational Electronics, Hyderabad, India. At UTS, he directed the Sydney Microwave Design Resource Centre and was the Associate Program Leader of the Cooperative Research Centre for Satellite Systems. He currently directs the Microwave and Wireless Technology Research Laboratory and is a core member of the University Research Centre for Health Technologies. His current teaching and research interests include wireless mobile communications, microwaves and antennas, smart antennas, and applications of microwave and wireless technology in medicine, and he has obtained many competitive research grants in these areas. $\mathrm{He}$ was a corecipient of the Priestly Memorial Award from the Institute of Radio and Electronic Engineers (IREE), Australia. He was a Member of the Organizing and Technical Program Committees of the IEEE Globecom'98, APMC 2000, International Symposium on Wireless Systems and Networks, 2003, and IASTED International Conference on Antennas, Radar, and Wave Propagation, for 2004 and 2005. 